\title{
Supporting Information: Integration of Methanol Aromatization with Light Hydrocarbon Aromatization Towards Increasing Aromatic Yields: Conceptual Process Designs and Comparative Analysis
}

\author{
Dan Zhang ${ }^{\dagger}$, Minbo Yang ${ }^{\dagger, *}$, Xiao Feng ${ }^{\dagger}$, Yufei Wang ${ }^{\ddagger}$ \\ ${ }^{\dagger}$ Shaanxi Key Laboratory of Energy Chemical Process Intensification, School of Chemical \\ Engineering and Technology, Xi'an Jiaotong University, No.28, Xianning West Road, Beilin \\ District, Xi’an, Shaanxi 710049, China \\ $\$$ State Key Laboratory of Heavy Oil Processing, China University of Petroleum (Beijing), No.18, \\ Fuxue Road, Changping District, Beijing 102249, China \\ *Corresponding author: yangmb@xjtu.edu.cn
}

The number of pages: 6

The number of tables: 5

Contents:

Table S1: The product specifications

Table S2: Operating parameters of key columns

Table S3: Input parameters for annual sales

Table S4: Input parameters for total annual production costs

Table S5: The parameters of LCA data for coal to methanol 
Table S1. The product specifications.

\begin{tabular}{|c|c|c|c|c|c|c|}
\hline & \multicolumn{6}{|c|}{ Products } \\
\hline & Benzene & Toluene & Xylene & $\mathrm{C}_{9}+$ aromatics & & Dry gas \\
\hline Items & \multicolumn{4}{|c|}{ Specifications $/ \mathrm{wt}^{0}{ }^{1}$} & Items & Specifications $/$ vol. $\%{ }^{2}$ \\
\hline Benzene & & $<0.05$ & & & & \\
\hline Toluene & 0.05 & & $<0.10$ & & & \\
\hline Xylene & & $<0.10$ & & $<0.05$ & $\mathrm{C}_{3}+$ & $<=3$ \\
\hline Non-aromatics & $<0.10$ & $<0.25$ & $<0.25$ & & & \\
\hline
\end{tabular}


Table S2. Operating parameters of key columns used in the process simulation. (In each cell, the first value represents the operating parameter at the top of a column, and the second value represents the operating parameter at the bottom of a column.)

\begin{tabular}{|c|c|c|c|c|c|c|c|c|c|}
\hline \multirow{9}{*}{ TIMTA } & \multirow{2}{*}{ Columns } & \multicolumn{2}{|c|}{$35 \%$} & \multicolumn{2}{|c|}{$55 \%$} & \multicolumn{2}{|c|}{$75 \%$} & \multicolumn{2}{|c|}{$95 \%$} \\
\hline & & Temperature $/{ }^{\circ} \mathrm{C}$ & Pressure $/ \mathrm{kPa}$ & Temperature $/{ }^{\circ} \mathrm{C}$ & Pressure $/ \mathrm{kPa}$ & Temperature $/{ }^{\circ} \mathrm{C}$ & Pressure $/ \mathrm{kPa}$ & Temperature/ ${ }^{\circ} \mathrm{C}$ & Pressure $/ \mathrm{kPa}$ \\
\hline & $\mathrm{T}-100$ & - & - & - & - & {$[86.0,201.5]$} & {$[600,650]$} & {$[84.6,200.7]$} & {$[600,650]$} \\
\hline & $\mathrm{T}-101$ & {$[73.7,140.1]$} & {$[120,150]$} & {$[73.7,139.5]$} & {$[120,150]$} & {$[85.8,138.9]$} & {$[120,150]$} & {$[85.8,138.0]$} & {$[120,150]$} \\
\hline & $\mathrm{T}-102$ & {$[116.4,159.0]$} & {$[120,150]$} & {$[116.4,159.0]$} & {$[120,150]$} & {$[116.4,159.0]$} & {$[120,150]$} & {$[116.4,159.1]$} & {$[120,150]$} \\
\hline & $\mathrm{T}-103$ & {$[146.3,183.7]$} & {$[120,150]$} & {$[146.3,183.7]$} & {$[120,150]$} & {$[146.3,183.6]$} & {$[120,150]$} & {$[146.3,183.6]$} & {$[120,150]$} \\
\hline & $\mathrm{T}-104$ & {$[-46.7,60.2]$} & {$[1700,1800]$} & {$[-47.5,62.7]$} & {$[1700,1800]$} & {$[-48.5,66.3]$} & {$[1700,1800]$} & {$[-51.6,69.9]$} & {$[1700,1800$} \\
\hline & T-105 & {$[39.8,164.6]$} & {$[100,100]$} & {$[33.1,163.0]$} & {$[100,100]$} & - & - & - & - \\
\hline & $\mathrm{T}-106$ & {$[85.7,258.7]$} & {$[50,50]$} & {$[86.1,258.7]$} & {$[50,50]$} & - & - & - & - \\
\hline \multirow{9}{*}{ PIMTA } & \multirow{2}{*}{ Columns } & \multicolumn{2}{|c|}{$35 \%$} & \multicolumn{2}{|l|}{$55 \%$} & \multicolumn{2}{|c|}{$75 \%$} & \multicolumn{2}{|c|}{$95 \%$} \\
\hline & & Temperature $/{ }^{\circ} \mathrm{C}$ & Pressure $/ \mathrm{kPa}$ & Temperature $/{ }^{\circ} \mathrm{C}$ & Pressure $/ \mathrm{kPa}$ & Temperature $/{ }^{\circ} \mathrm{C}$ & Pressure/kPa & Temperature/ ${ }^{\circ} \mathrm{C}$ & Pressure $/ \mathrm{kPa}$ \\
\hline & $\mathrm{T}-100$ & - & - & - & - & {$[80.8,201.5]$} & {$[600,650]$} & {$[78.9,200.7]$} & {$[600,650]$} \\
\hline & $\mathrm{T}-101$ & {$[73.7,140.2]$} & {$[120,150]$} & {$[73.7,139.4]$} & {$[120,150]$} & {$[85.8,138.9]$} & {$[120,150]$} & {$[85.8,138.0]$} & {$[120,150]$} \\
\hline & $\mathrm{T}-102$ & {$[116.4,159.0]$} & {$[120,150]$} & {$[116.4,159.0]$} & {$[120,150]$} & {$[116.4,159.0]$} & {$[120,150]$} & {$[116.4,159.1]$} & {$[120,150]$} \\
\hline & $\mathrm{T}-103$ & {$[146.3,183.7]$} & {$[120,150]$} & {$[146.3,183.7]$} & {$[120,150]$} & {$[146.3,183.6]$} & {$[120,150]$} & {$[146.3,183.6]$} & {$[120,150]$} \\
\hline & T-104 & {$[-46.7,59.8]$} & {$[1700,1800]$} & {$[-47.5,62.2]$} & {$[1700,1800]$} & {$[-48.6,65.5]$} & {$[1700,1800]$} & {$[-51.5,71.0]$} & {$[1700,1800$} \\
\hline & $\mathrm{T}-105$ & {$[37.1,166.0]$} & {$[100,100]$} & {$[30.5,164.5]$} & {$[100,100]$} & - & - & - & - \\
\hline & $\mathrm{T}-106$ & {$[87.9,258.7]$} & {$[50,50]$} & {$[87.8,258.7]$} & {$[50,50]$} & - & - & - & - \\
\hline
\end{tabular}


Table S3. Input parameters for annual sales

\begin{tabular}{ccccc}
\hline Items & Values & Units & Notes & Sources \\
\hline Benzene & 1046.18 & $\$ / \mathrm{t}$ & $\mathrm{a}$ & 3 \\
Toluene & 1184.08 & $\$ / \mathrm{t}$ & $\mathrm{a}$ & 4 \\
Xylene & 1270.72 & $\$ / \mathrm{t}$ & $\mathrm{a}$ & 5 \\
$\mathrm{C}_{9}+$ & 938.60 & $\$ / \mathrm{t}$ & $\mathrm{a}$ & 6 \\
Dry gas & 2.15 & $10^{-5} \$ / \mathrm{kJ}$ & $\mathrm{b}$ & 7 \\
\hline
\end{tabular}

${ }^{a}$ The annual sales are calculated by the average prices of Chinese market in 2018 .

${ }^{b}$ The dry gas price is gotten by the price of natural gas based on heat value.

Table S4. Input parameters for total annual production costs

\begin{tabular}{ccccc}
\hline Items & Values & Units & Notes & Sources \\
\hline Methanol & 382.66 & $\$ / \mathrm{t}$ & $\mathrm{a}$ & 8 \\
Sulfolane & 2000 & $\$ / \mathrm{t}$ & $\mathrm{a}$ & 9 \\
LP Steam & 2.00 & $10^{-6} \$ / \mathrm{kJ}$ & $\mathrm{b}$ & - \\
MP Steam & 2.00 & $10^{-6} \$ / \mathrm{kJ}$ & $\mathrm{b}$ & - \\
HP Steam & 3.00 & $10^{-6} \$ / \mathrm{kJ}$ & $\mathrm{b}$ & - \\
SHP Steam & 4.00 & $10^{-6} \$ / \mathrm{kJ}$ & $\mathrm{b}$ & - \\
Cooling Water & 0.21 & $10^{-6} \$ / \mathrm{kJ}$ & $\mathrm{b}$ & - \\
Refrigerant 4 & 8.98 & $10^{-6} \$ / \mathrm{kJ}$ & $\mathrm{b}$ & - \\
Electricity & 16.00 & $10^{-6} \$ / \mathrm{kJ}$ & $\mathrm{b}$ & - \\
HP steam generation & 3.00 & $10^{-6} \$ / \mathrm{kJ}$ & $\mathrm{b}$ & - \\
$N U$ & 25 & - & $\mathrm{c}$ & - \\
$S U$ & 12480 & $\$ /$ operator/yr & $\mathrm{c}$ & - \\
$N A$ & 25 & - & $\mathrm{d}$ & - \\
$S A$ & 24960 & $\$ /$ operator/yr & $\mathrm{d}$ & - \\
$N C$ & 25 & - & $\mathrm{e}$ & - \\
$S C$ & 31200 & $\$ /$ operator/yr & $\mathrm{e}$ & - \\
\hline
\end{tabular}

${ }^{a}$ The feedstock costs are calculated by the average prices of Chinese market in 2018.

${ }^{\mathrm{b}}$ The costs of utilities are estimated by Aspen Process Economic Analyzer V10.0.

${ }^{\mathrm{c}} N U$ is the number of operators; $S U$ is the annual salary of per operator.

${ }^{\mathrm{d}} N A$ is the number of operators; $S A$ is the annual salary of per operator for technical assistance.

${ }^{\mathrm{e}} N C$ is the number of operators for control laboratory; $S C$ is the annual salary of per operator for control laboratory. 
Table S5. The parameters of LCA data for coal to methanol ${ }^{10}$

\begin{tabular}{cccccc}
\hline Inputs & Values & Units & Outputs & Values & Units \\
\hline Coal acquisition & & & & & \\
Electricity & 1.92 & $\mathrm{MJ}$ & Hard coal & 13.7 & $\mathrm{~kg}$ \\
Fresh water & 6.85 & $\mathrm{~kg}$ & Gangue & 1.52 & $\mathrm{~kg}$ \\
Hard coal & 15.22 & $\mathrm{~kg}$ & $\mathrm{CH}_{4}$ & $7.59 \times 10^{-2}$ & $\mathrm{~kg}$ \\
Oil & $1.52 \times 10^{-2}$ & $\mathrm{~kg}$ & & & \\
Methanol production & & & & & \\
HP steam & 17.58 & $\mathrm{~kg}$ & Methanol & 9.93 & $\mathrm{~kg}$ \\
Electricity & 69.11 & $\mathrm{MJ}$ & Slag & 1.49 & $\mathrm{~kg}$ \\
Air & 43.18 & $\mathrm{~kg}$ & MP nitrogen & 15.13 & $\mathrm{~kg}$ \\
Water & 1472 & $\mathrm{~kg}$ & $\mathrm{CO}_{2}$ & $1.73 \times 10^{-2}$ & $\mathrm{~kg}$ \\
Hard coal & 13.7 & $\mathrm{~kg}$ & Sulphur & 0.298 & $\mathrm{~kg}$ \\
Ammonia & 0.298 & $\mathrm{~kg}$ & Flue gas & 0.695 & $\mathrm{~kg}$ \\
NaOH $(10 \%)$ & $9.93 \times 10^{-3}$ & $\mathrm{~kg}$ & Effluents & 2.64 & $\mathrm{~kg}$ \\
\hline
\end{tabular}




\section{References}

(1) Xu, C. E. The Technologies of Catalytic Reforming. China Petrochemical Press: Beijing, 2014; pp 952-958.

(2) HCBBS, https://bbs.hcbbs.com/thread-2220468-1-1.html (accessed Sept 10, 2018).

(3) PPI, http://ben.100ppi.com/ (accessed Nov 27, 2018).

(4) OilChem, http://chem.oilchem.net/chemical/toluene.shtml (accessed Nov 24, 2018).

(5) ChemCP, http://www.chemcp.com/news/201810/900678.asp (accessed Nov 28, 2018).

(6) OilChem, http://price.oilchem.net/dmPrice/listProduct.lz?pName=C9\&webFlag=1\&hndz=0 (accessed Nov 29, 2018).

(7) OilChem, http://oil.oilchem.net/oil/NG.shtml (accessed Dec 01, 2018).

(8) OilChem, http://chem.oilchem.net/chemical/methanol.shtml (accessed Nov 25, 2018).

(9) Alibaba, https://www.alibaba.com/showroom/price-sulfolane.html (accessed Jan 27, 2020)

(10) Chen, Z., Shen, Q., Sun, N., Wei, W. Life cycle assessment of typical methanol production routes: The environmental impacts analysis and power optimization. J. Clean. Prod. 2019, 220, 408-416. https://doi.org/10.1016/j.jclepro.2019.02.101. 